\title{
Inhibition or deletion of the lipopolysaccharide receptor Toll-like receptor- 4 confers partial protection against lipid-induced insulin resistance in rodent skeletal muscle
}

\author{
M. S. Radin • S. Sinha • B. A. Bhatt • N. Dedousis • \\ R. M. O'Doherty
}

Received: 31 July 2007 / Accepted: 23 August 2007 / Published online: 1 December 2007

(C) Springer-Verlag 2007

\begin{abstract}
Aims/hypothesis A role for increased activity of the innate immune system in the pathogenesis of insulin resistance is supported by a number of studies. The current study assessed the potential role of the lipopolysaccharide receptor known as Toll-like receptor-4 (TLR-4), a component of the innate immune system, in mediating lipidinduced insulin resistance in skeletal muscle.

Methods The effects of TLR-4 inhibition/deletion on lipidinduced insulin resistance was determined in skeletal muscle of TLR-4 null mice in vivo and in rat L6 myotubes in vitro. Results In mice, acute hyperlipidaemia induced skeletal muscle insulin resistance, but a deletion of TLR-4 conferred significant protection against these effects. In L6 myotubes, inhibition of TLR-4 activity substantially reduced the capacity of the saturated fatty acid palmitate to induce insulin resistance. Importantly, palmitate activated the nuclear factor $\kappa \mathrm{B}(\mathrm{NF} \kappa \mathrm{B})$ pathway in L6 myotubes and mouse skeletal muscle, and these effects were blocked by inhibition of TLR-4 in L6 myotubes and absence of TLR-4 in
\end{abstract}

M. S. Radin $\cdot$ S. Sinha $\cdot$ B. A. Bhatt $\cdot$ N. Dedousis $\cdot$

R. M. O'Doherty

Department of Medicine, Division of Endocrinology,

University of Pittsburgh,

Pittsburgh, PA, USA

R. M. O’Doherty

Department of Molecular Genetics and Biochemistry,

University of Pittsburgh,

Pittsburgh, PA, USA

R. M. O’Doherty $(\bowtie)$

Division of Metabolism/Endocrinology,

University of Pittsburgh Medical Center,

E1112 Biomedical Science Tower, Lothrop Street,

Pittsburgh, PA 15261, USA

e-mail: odohertyr@dom.pitt.edu skeletal muscle. Furthermore, inhibition of the NFkB pathway downstream of TLR-4 in L6 myotubes also protected against the induction of insulin resistance by palmitate.

Conclusions/interpretation Inhibition or absence of TLR-4 confers protection against the detrimental effects of lipids on skeletal muscle insulin action, and these effects are associated with a prevention of the activation of the NFKB pathway by lipids. Importantly, inhibition of the NFkB pathway in myotubes downstream of TLR-4 also protects against lipid-induced insulin resistance, suggesting a mechanism by which reduced TLR-4 activity confers beneficial effects on insulin action.

Keywords Immune system · Insulin resistance - Lipids · Toll-like receptor $\cdot$ NF $\kappa$ B $\cdot$ Skeletal muscle

$\begin{array}{ll}\text { Abbreviations } \\ \text { ASA } & \text { acetylsalicylate } \\ \text { GIR } & \text { glucose infusion rate } \\ \text { I } B \alpha & \text { inhibitor of } \kappa \text { B } \alpha \\ \text { IKK } & \text { inhibitor of } \kappa \text { B kinase } \\ \text { LPS } & \text { lipopolysaccharide } \\ \text { MEM } \alpha & \text { Minimum Essential Medium } \alpha \\ \text { NFkB } & \text { nuclear factor } \kappa \text { B } \\ \text { PI3-kinase } & \text { phosphatidylinositol 3-kinase } \\ \text { PI(3)P } & \text { phosphatidylinositol 3-phosphate } \\ \text { TIP } & \text { TIRAP inhibitor peptide } \\ \text { TLR } & \text { Toll-like receptor }\end{array}$

Introduction

Insulin resistance is a characteristic feature of obesity and is a primary risk factor for the development of type 2 diabetes. 
Despite intensive efforts, the pathogenesis of insulin resistance is not well understood, although a number of biochemical mechanisms have been proposed. In short, defects in insulin signalling pathways, alterations in the production and/or activity of a variety of adipokines and cytokines, and dyslipidaemia/dysregulated lipid metabolism may each, separately or in combination, contribute to the development of insulin resistance. More recently, increased activity of the innate immune system and inflammatory pathways have been suggested as important mechanisms of insulin resistance [1-17]. Markers of inflammation are increased in obesity and type 2 diabetes [18-20], and macrophage infiltration of adipose tissue occurs in insulinresistant states $[4,5,7]$. Importantly, an increase in the activity of the proinflammatory nuclear factor $\mathrm{KB}(\mathrm{NF} \kappa \mathrm{B})$ pathway $[13,16]$ or an elevation of adipose macrophage infiltration [6] induces insulin resistance, while a decrease in the activity of the NFKB pathway $[1,10,12,15]$ or inhibition of adipose macrophage infiltration $[2,3]$ protects against the development of insulin resistance induced by obesity and lipids. The elements of the innate immune system that may play a role in the pathogenesis of insulin resistance, the tissues targeted, and the mechanisms involved in their activation remain unclear.

Toll-like receptors (TLRs) are a family of receptors whose activation plays a critical role in mediating the innate immune response in mammals by protecting against viral, fungal, protozoan and bacterial infections [21-24]. Specifically, TLRs in macrophages are activated by specific conserved pathogen recognition patterns, such as lipopolysaccharide (LPS), single-stranded RNA, peptidoglycans and flagellin $[25,26]$. To date, approximately 12 TLRs have been identified, each of which associates with particular ligands. Of most relevance to the current study is TLR-4, which is activated by LPS (possibly through a lauric acid moiety) [21], and saturated fatty acids [27, 28]. A proximal target of TLR-4 activity is the NFKB pathway, which itself has been implicated as a mechanism of insulin resistance $[1,10,12$, $13,15,16]$ and is activated by lipids $[8,11,13,14]$. Although the majority of work has focused on TLR-4 function in macrophages, this receptor is also expressed in other tissues [29, 30], although its function in these is unclear. In a recent study, Shi et al. [17] demonstrated that an absence of TLR-4 protects against the detrimental effects of obesity and lipids on insulin resistance. However, this study emphasised the role of adipose tissue TLR-4 in these effects, and did not address the potential role of TLR-4 in skeletal muscle, the predominant insulin-sensitive tissue in the body. Based on this, the aim of the current study was to investigate the role of skeletal muscle TLR-4 in the induction of insulin resistance by lipids. The data demonstrate that (1) TLR-4 is expressed in skeletal muscle and is activated by lipids; (2) inhibition or absence of TLR-4 reduces the capacity of lipids to induce skeletal muscle insulin resistance; (3) TLR-4 mediates lipid-induced activation of the $\mathrm{NF} \kappa \mathrm{B}$ pathway in skeletal muscle; and (4) inhibition of the NFKB pathway downstream of TLR-4 protects against lipid-induced insulin resistance.

\section{Methods}

Materials Minimum Essential Medium $\alpha(\operatorname{MEM} \alpha)$ and penicillin/streptomycin were obtained from Invitrogen (Carlsbad, CA, USA). Fetal bovine serum was from Cambrex Bio Science (Walkersville, MD, USA). Trypsin/ EDTA was from Mediatech (Herndon, VA, USA). Palmitic acid was obtained from Nu-Chek (Elysian, MN, USA). 2Deoxy-D-[1,2- $\left.{ }^{3} \mathrm{H}\right]$ glucose and $\gamma-\left[{ }^{32} \mathrm{P}\right]$ ATP were obtained from PerkinElmer Life (Boston, MA, USA). Polymyxin was obtained from Sigma (St Louis, MO, USA). TIRAP inhibitor peptide (TIP) was obtained from CalBiochem/ EMD Biosciences (La Jolla, CA, USA). Insulin (Humulin) and dextrose were obtained from Eli Lilly (Indianapolis, IL, USA) and Hospira (Lake Forest, IL, USA), respectively. Lipid (Liposyn II) and heparin were obtained from Abbott Laboratories (Chicago, IL, USA). Reagents for PAGE were from Bio-Rad (Hercules, CA, USA).

Animal care and maintenance Male mice [C57/B10 ScN (TLR-4 null) and C57/B10 ScSn (wild-type)] were purchased from Jackson Laboratories (Bar Harbor, ME, USA). After arrival, mice were maintained on a constant $12 \mathrm{~h}$ light $/ 12 \mathrm{~h}$ dark cycle with free access to water and standard chow. All the experimental procedures were approved by the Institutional Animal Care and Use Committee (IACUC) of the University of Pittsburgh.

Implantation of chronic indwelling catheters Animals were anaesthetised with Nembutal $(60-75 \mathrm{mg} / \mathrm{kg})$. Catheters (PE-10, Intramedic; Becton Dickinson, Sparks, MD, USA) were introduced into the left carotid artery (advanced to the aortic arch) and the right jugular vein (advanced to the right atrium). The catheters were exteriorised at the back of the neck, filled with a sterile 3:1 glycerol/heparin mix and plugged. The wounds were closed with sutures and treated with Betadine (Purdue Frederick, Stamford, CT, USA). The animals were treated subcutaneously with ketoprofen $(2 \mathrm{mg} / \mathrm{kg}$ ) immediately and $24 \mathrm{~h}$ after surgery and their recovery was monitored, with special attention given to food intake, weight gain and healing of wounds. Animals were allowed 4-6 days to recover and only those that had achieved $>85 \%$ of their pre-surgery weight were used in the studies.

Lipid infusions, hyperinsulinaemic clamps and skeletal muscle glucose uptake All animals were fasted for $5 \mathrm{~h}$ 
prior to experiments. At $0 \mathrm{~h}$, lipid/heparin (Liposyn II, $5 \mathrm{ml}$ $\mathrm{kg}^{-1} \mathrm{~h}^{-1}$ and heparin $6 \mathrm{U} / \mathrm{h}$ ), insulin (Humulin, $15 \mathrm{mU} \mathrm{kg}^{-1}$ $\mathrm{min}^{-1}$ or $4 \mathrm{mU} \mathrm{kg}^{-1} \mathrm{~min}^{-1}$ ) and glucose infusions commenced and continued for $240 \mathrm{~min}$. During this period the glucose infusion rate (GIR) was adjusted to maintain plasma glucose concentrations at euglycaemia $(\sim 6.7 \mathrm{mmol} / \mathrm{l})$. The $15 \mathrm{mU} \mathrm{kg}{ }^{-1} \mathrm{~min}^{-1}$ insulin infusion rate used ensured that hepatic glucose output was completely suppressed. In these experiments the GIRs from $t=30-60 \mathrm{~min}$ and $t=60$ $240 \mathrm{~min}$ of the clamp were used as indices of insulin sensitivity, as previously described [31-33]; $t=30-60 \mathrm{~min}$ represents a time period in the clamp prior to the induction of insulin resistance by lipids. For the direct assessment of skeletal muscle glucose uptake in vivo, a bolus injection of 2-deoxy-D-[1,2- $\left.{ }^{3} \mathrm{H}\right]$ glucose $\left(55.5 \times 10^{4} \mathrm{~Bq}[15 \mu \mathrm{Ci}]\right)$ was administered at $t=225 \mathrm{~min}$ of a $4 \mathrm{mU} \mathrm{kg}^{-1} \mathrm{~min}^{-1}$ insulin clamp, with lipid infusions and blood samples taken at 2.5, 5.0, 10.0, 15.0 and $25.0 \mathrm{~min}$ after the injection. Subsequently, animals were killed and skeletal muscle taken for analysis. Skeletal muscle and plasma were analysed as previously described [34].

High-fat feeding and insulin tolerance tests Mice were placed on standard chow or high-fat (45\% of calories from fat, TD96001; Harlan Teklad, Madison, WI, USA) diets for 21 weeks, and weight gain monitored. For insulin tolerance tests a baseline blood glucose measurement was made in ad libitum fed mice, and an i.p. insulin bolus $(0.75 \mathrm{U} / \mathrm{kg})$ was then administered. The blood glucose concentration was monitored every $15 \mathrm{~min}$ over $120 \mathrm{~min}$ by tail vein bleeding.

L6 myotube experimental design In experiments to test for the functionality of TLR-4, L6 myotubes were exposed to $2 \mu \mathrm{g} / \mathrm{ml}$ LPS (Sigma) for $2 \mathrm{~h}$ or LPS plus polymyxin $(20 \mu \mathrm{g} / \mathrm{ml})$ or LPS plus TIP $(40 \mu \mathrm{mol} / \mathrm{l})$ in MEM $\alpha$ containing $25 \mathrm{mmol} / 1$ glucose. When used, inhibitors of LPS function (polymyxin, which acts by sequestering/ precipitating LPS) or TLR-4 signalling (TIP) were added $1 \mathrm{~h}$ prior to LPS. For experiments addressing the effects of palmitate on insulin sensitivity or NFKB activity, L6 myotubes were exposed for $6 \mathrm{~h}$ to palmitate $(0.4 \mathrm{mmol} / \mathrm{l})$ conjugated to fatty acid-free BSA in MEM $\alpha$ containing $25 \mathrm{mmol} / \mathrm{l}$ glucose, conditions that induce insulin resistance and activation of the NFKB pathway, as demonstrated previously [13]. Subsequently, insulin-stimulated glucose uptake and DNA binding of NFKB were assessed as described below. Where used, inhibitors of TLR-4 signalling (TIP, $40 \mu \mathrm{mol} / \mathrm{l}$ ) or inhibitor of $\kappa \mathrm{B}$ kinase (IKK; acetylsalicylate [ASA], $5 \mathrm{mmol} / \mathrm{l}$ ) were added $1 \mathrm{~h}$ prior to the addition of palmitate.

Isolated soleus muscle experimental design For experiments requiring mouse skeletal muscle, mice were anaes- thetised with Nembutal and the soleus muscle was isolated from both hind limbs and immediately placed in DMEM receiving continuous oxygenation in a shaking water bath at $29^{\circ} \mathrm{C}$. One muscle from each animal was exposed to either LPS or BSA/palmitate. The contralateral muscle was exposed to vehicle or BSA alone. All muscles were subsequently flash frozen in liquid nitrogen and stored at $-70^{\circ} \mathrm{C}$ until analysis of inhibitor of $\mathrm{\kappa} \mathrm{B} \alpha(\mathrm{I} \kappa \mathrm{B} \alpha)$ and tubulin by immunoblot. For each condition in each experiment, muscles from three animals were used. Muscles from the same condition in each experiment were pooled for analysis.

Palmitate and LPS solutions Stock palmitate solutions were prepared by conjugating palmitic acid with fatty acid-free BSA (Sigma) as previously described [13]. A stock LPS solution was prepared in sterile deionised water.

Nuclear protein extracts and EMSAs Nuclear protein extracts were prepared and EMSAs for the analysis of DNA binding of $N F \kappa B$ were performed as previously described [13].

L6 cell 2-deoxy-D- $\left[1,2-{ }^{3} H\right]$ glucose uptake 2-Deoxy-D$\left[1,2-{ }^{3} \mathrm{H}\right]$ glucose uptake was determined as previously described [13].

Tlr-4 gene expression Expression of Tlr-4 mRNA in L6 myotubes was assessed by RT-PCR. Two sets of oligonucleotides 21 bases long (Integrated DNA Technologies, Coralville, IA, USA) derived from the Rattus norvegicus Tlr-4 mRNA sequence (accession no. NM019178) were used for the PCR (set no. 1: forward 5'-GATGAAACATCAGAGGAA GAA-3', reverse 5'-TTGCCTGAGTTGCTTAATTCA-3'; set no. 2: forward 5'-TTATTAAACATGAGTCACAAC-3', reverse 5'TCATTTGTTCAACATTCACCA-3'). For mice, the oligonucleotides used were derived from the Mus musculus Tlr-4 mRNA sequence (accession no. NM021297; forward 5'-AG CTACTTTACCTTGACATCT-3', reverse 5'-GATTGTTGT GACTATTTAATA-3').

Quantitative RT-PCR Total RNA was isolated from skeletal muscle using Tri-reagent (Molecular Research Center, Cincinnati, OH, USA) according to the manufacturer's instructions. For the quantitative real-time PCR analyses, $2.5 \%$ of the total RT reaction was used as input for PCR using SYBR Green Master Mix (Applied Biosystems, Foster City, CA, USA), or Bio-Rad iTaq SYBR Green Supermix with ROX. The relative abundance of mRNA was determined by comparative cycle threshold analysis according to Applied Biosystems User Bulletin no. 2.

Phosphatidylinositol 3-kinase activity, Akt, GLUT4 and $I \kappa B \alpha$ Phosphatidylinositol 3-kinase (PI3-kinase) activity 
was assayed according to a standard protocol. Briefly, immunoprecipitation of active PI3-kinase was achieved by incubating $250 \mu \mathrm{g}$ of skeletal muscle protein (derived from insulin clamp studies) with $3 \mu \mathrm{g}$ of $\alpha$-IRS-1 antibody (Upstate, New York, NY, USA) for $2 \mathrm{~h}$ followed by the addition of Protein A Sepharose for $1.5 \mathrm{~h}$ (Amersham Biosciences, Piscataway, NJ, USA). The immune complexes were incubated for $10 \mathrm{~min}$ at $22^{\circ} \mathrm{C}$ with PI $(10 \mu \mathrm{g}$; Avanti Polar Lipids, Alabaster, AL, USA) in the presence of $50 \mu \mathrm{mol} / 1\left[\gamma_{-}{ }^{32} \mathrm{P}\right]$ ATP $\left[18.5 \times 10^{4} \mathrm{~Bq}(5 \mu \mathrm{Ci})\right.$, PerkinElmer Life]. $\left[{ }^{32} \mathrm{P}\right]$-containing phosphatidylinositol 3-phosphate [PI (3)P] was separated by thin-layer chromatography (TLC) and was quantified by scraping the PI(3)P spot from the TLC plate, followed by scintillation counting. Total Akt (Cell Signaling, Beverly, MA, USA) and phosphorylated

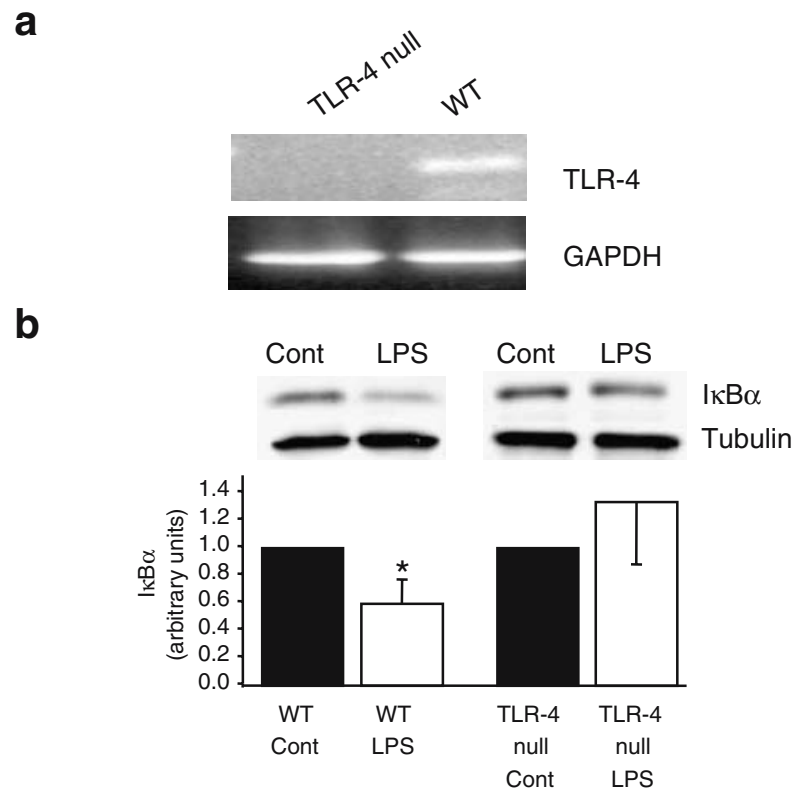

\section{C}

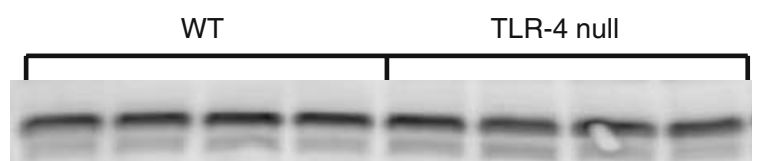

Fig. 1 Expression of Tlr-4 and effect of LPS on activation of skeletal muscle TLR-4. a Expression of Tlr-4 mRNA in skeletal muscle from wild-type (WT) and TLR-4 null mice. b Soleus muscle from a wildtype or TLR-4 null mouse was exposed to LPS for $2 \mathrm{~h}$. The contralateral soleus muscle from the same animal was exposed to vehicle for a similar length of time. For each condition in each experiment, muscles from three animals were used and pooled after exposures for analysis of $\operatorname{IkB} \alpha$ and tubulin by immunoblot. Representative autoradiographs are shown. Quantification of the complete data set is shown below the autoradiograph. For the purposes of quantification, $\operatorname{I} \mathrm{B} \alpha$ values were normalised to tubulin values, and levels of $\operatorname{I} \mathrm{B} \alpha$ in control skeletal muscle were arbitrarily set to a value of $1 .{ }^{*} p<0.05$ vs wild-type control (cont) muscle. $n=9$ animals per group. Results are presented as means \pm SE. $\mathbf{c}$ Basal I $\mathrm{B} \alpha$ levels in wild-type and TLR-4 null mouse skeletal muscle as determined by immunoblotting
Akt (serine-473; Cell Signaling), IкB $\alpha$ (Santa Cruz Biotechnology, Santa Cruz, CA, USA) and $\alpha$-tubulin (Santa Cruz Biotechnology) were measured with commercially available antibodies. The GLUT4 antibody was obtained from the National Institutes of Health (Bethesda, MD, USA). All proteins were measured using a standard immunoblot protocol. Quantification of protein levels was accomplished by densitometric analysis of autoradiographs.

Tissue and plasma measurements Tissue triacylglycerols were determined as described previously [35] using the GPO-triglyceride kit and Lintrol lipids as standard (Sigma). Plasma triacylglycerols were measured using the same kit. Insulin was measured using a rat-specific RIA kit (Linco Research, St Charles, MO, USA). Plasma glucose concentrations were determined using a glucose analyser (Glucose 2; Beckman Coulter, Fullerton, CA, USA).

Statistical methods All results are expressed as means \pm SE. Statistical significance was determined by paired or unpaired

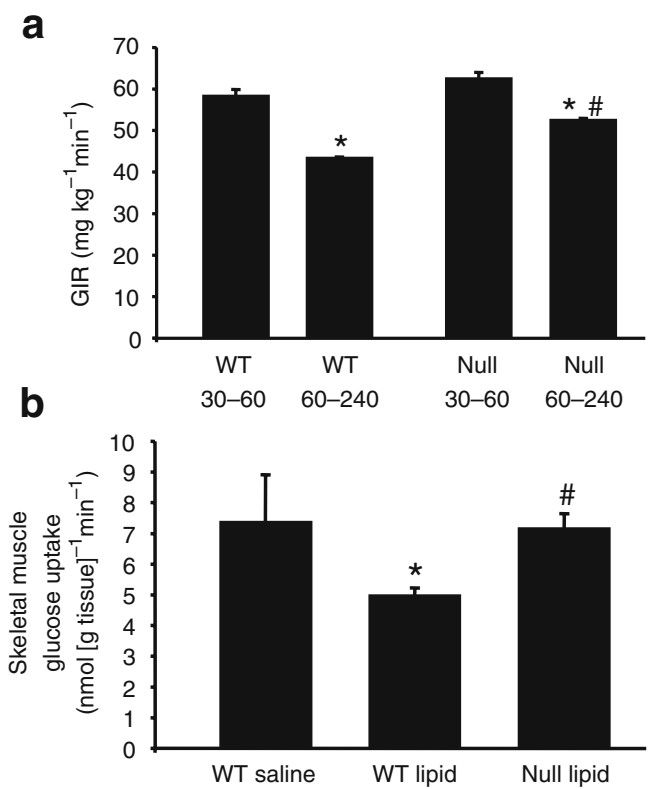

Fig. 2 Effects of TLR-4 deletion on lipid-induced insulin resistance. a Wild-type (WT) and TLR-4 null mice received insulin, glucose and lipid/heparin infusions for $240 \mathrm{~min}$ as described in Methods. Mean glucose infusion rates are shown for the period $t=30-60 \mathrm{~min}(30-60)$ and $t=60-240 \mathrm{~min}(60-240)$. * Significant difference $(p<0.05)$ between $60-240$ and 30-60. Significant difference $(p<0.05)$ between null and wild-type. b Wild-type and TLR-4 null mice received insulin, glucose and lipid/heparin infusions; the wild-type control mice received a saline infusion in place of lipid/heparin. At $t=225 \mathrm{~min}$ a bolus injection of 2-deoxy-D-[1,2- H]glucose [55.5×10 Bq $(15 \mu \mathrm{Ci})]$ was administered and blood samples taken at 2.5, 5.0, 10.0, 15.0 and $25.0 \mathrm{~min}$ after the injection. Animals were killed, tissues taken, and skeletal muscle glucose uptake calculated. *Significant difference $(p<0.05)$ between lipid/heparin infused and saline infused. Significant difference $(p<0.05)$ between null and wild-type. Results are presented as means \pm SE. $n=4-5$ animals per group 
Student's $t$ tests where appropriate using Microsoft Excel (Microsoft, Seattle, WA, USA). Statistical significance was assumed at a $p$ value of $<0.05$.

\section{Results}

TLR-4 is expressed and is functional in mouse skeletal muscle To begin to address a potential role for skeletal muscle TLR-4 in lipid-induced insulin resistance, we first determined whether $T l r-4$ is expressed in skeletal muscle. Expression of $T l r-4$ mRNA in soleus muscle of wild-type mice was readily detectable, but was absent in TLR-4 null mice (Fig. 1a). We next determined whether TLR-4 is functional in skeletal muscle by determining the capacity of LPS, the TLR-4 ligand [21], to activate the NFKB pathway, the proximal target of TLR activity [22, 24, 25]. Exposure of isolated soleus muscle to LPS increased the activity of the NFkB pathway ( $p=0.02$, Fig. $1 b)$, as measured by decreases in the levels of IKB $\alpha$. Furthermore, the absence of TLR-4 prevented the activation of the NFKB pathway by LPS in soleus muscle (Fig. 1b). Basal skeletal muscle IkB $\alpha$ levels were similar in wild-type and TLR-4 null animals (Fig. 1c). Taken together, these data demonstrate that TLR-4 is produced and is functional in skeletal muscle.

An absence of TLR-4 protects against lipid-induced insulin resistance in skeletal muscle in vivo We next determined

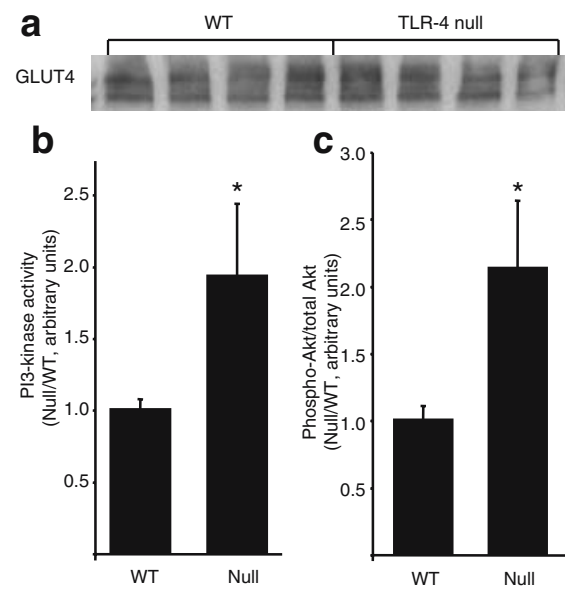

Fig. 3 Skeletal muscle GLUT4, PI3-kinase activity and Akt phosphorylation in lipid-infused TLR-4 null and wild-type (WT) mice. Wild-type and TLR-4 null mice received insulin, glucose and lipid/ heparin infusions for $240 \mathrm{~min}$ as described in Methods. Subsequently, animals were anaesthetised and skeletal muscle was isolated. GLUT4 (a), PI3-kinase (b), and Akt phosphorylation (c) were then measured as described in Methods. Parts $\mathbf{b}$ and $\mathbf{c}$ show the quantification of the total data sets as measured by scintillation counting of $\mathrm{PI}(3) \mathrm{P}$ production (PI3-kinase) or densitometry of autoradiographs (Akt). For Akt analysis, phospho-AKT was corrected to total Akt. $n=4-5$ in each group. *Statistical significance $(p<0.05)$. Results are presented as means $\pm \mathrm{SE}$

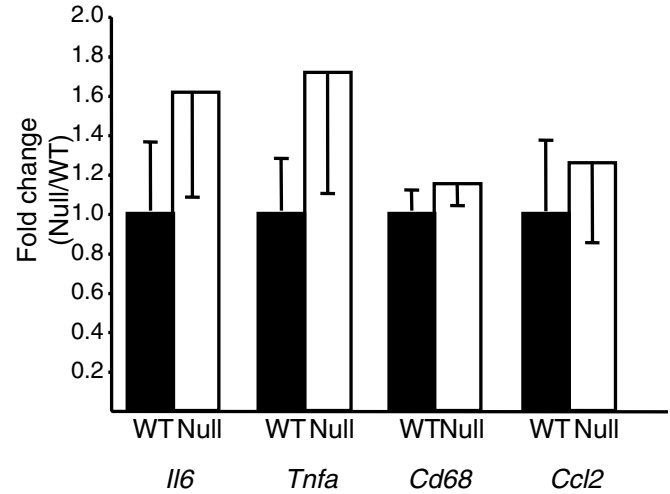

Fig. 4 Skeletal muscle inflammatory gene expression in lipid-infused TLR-4 null and wild-type (WT) mice. Skeletal muscle inflammatory gene expression from lipid-infused wild-type and TLR-4 null mice was analysed by real-time quantitative PCR as described in Methods. For the purposes of quantification, gene expression values were normalised to $\alpha$-tubulin mRNA values, and levels of test mRNAs in wildtype skeletal muscle were arbitrarily set to a value of $1 . n=4-5$ in each group. Results are presented as means $\pm \mathrm{SE}$

whether TLR-4 plays a role in mediating lipid-induced skeletal muscle insulin resistance in vivo. We assessed the effects of a $4 \mathrm{~h}$ lipid infusion on skeletal muscle glucose uptake during a hyperinsulinaemic-euglycaemic clamp in TLR-4 null and wild-type mice (Fig. 2). A lipid infusion decreased the GIR required to maintain euglycaemia in both wild-type and TLR-4 null mice. However, the GIR was significantly elevated in TLR-4 null mice compared with wild-type mice ( $p<0.001$, Fig. 2a), suggesting greater insulin sensitivity in TLR-4 null mice. This was confirmed by direct measurements of skeletal muscle glucose uptake (Fig. 2b). Thus, the capacity of insulin to stimulate muscle glucose uptake in lipid-infused TLR-4 null mice was increased $\sim 40 \%$ compared with lipid-infused wild-type mice $(p<0.01$ for lipid-infused TLR-4 null vs lipid-infused wild-type). Total GLUT4 levels were similar in wild-type and TLR-4 null mice (Fig. 3a). However, both IRS-1-associated PI3-kinase activity (Fig. 3b) and Akt phosphorylation (Fig. 3c) were increased in TLR-4 null mice compared with wild-type mice. These improvements in insulin signalling were not correlated with alterations in skeletal muscle expression of the genes encoding the inflammatory markers IL-6 (IlO), TNF- $\alpha$ (Tnfa), MCP-1 (Ccl2) or CD68 (Cd68) (Fig. 4).

Inhibition of TLR-4 function in L6 myotubes decreases palmitate-induced insulin resistance To further address the potential role and mechanisms of TLR-4-induced insulin resistance in skeletal muscle we investigated lipid-induced insulin resistance and TLR-4 function in L6 myotubes. We first determined whether Tlr-4 is expressed and the protein product is functional in L6 cells. Expression of Tlr-4 mRNA was confirmed in L6 myotubes (Fig. 5a). Exposure of L6 myotubes to LPS increased the activity of the NFKB pathway, as measured by increased DNA binding of nuclear 
a

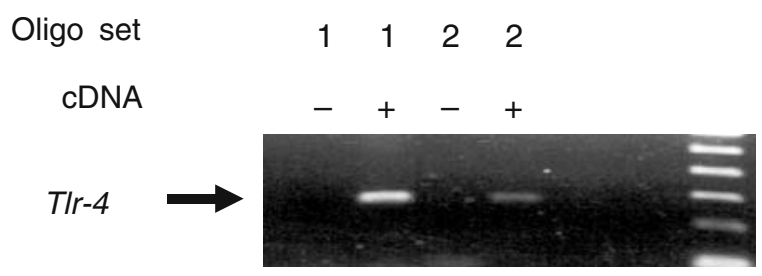

b

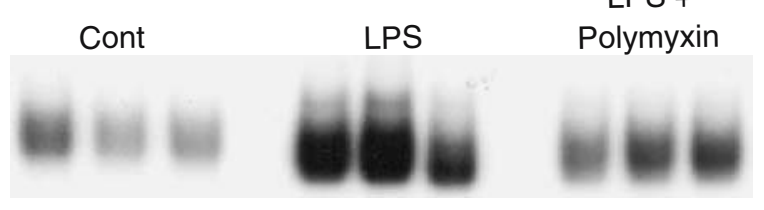

C

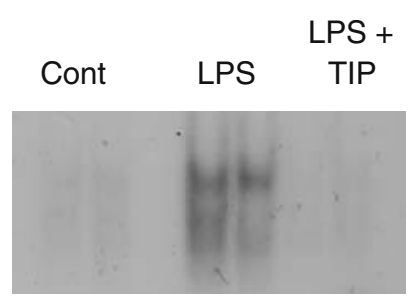

Fig. 5 Expression of $T l r-4$ and effect of LPS on activation of L6 myotube TLR-4. a Tlr-4 mRNA expression in L6 myotubes. Two different sets of oligonucleotides (set 1 and set 2) were used to assess expression as detailed in Methods. b L6 myotubes were exposed to vehicle (cont), LPS, or LPS plus polymyxin for $2 \mathrm{~h}$ as described in Methods. Subsequently, nuclear extracts were prepared and DNA binding of NFKB was determined. Representative EMSAs of three independent experiments run in duplicate or triplicate are shown. c L6 myotubes were exposed to vehicle (cont), LPS or LPS plus TIP for $2 \mathrm{~h}$. Subsequently, nuclear extracts were prepared and DNA binding of $\mathrm{NF} \kappa \mathrm{B}$ was determined. Note that TIP was added to cells $1 \mathrm{~h}$ prior to the addition of LPS. Representative EMSAs of three independent experiments for each condition run in duplicate or triplicate are shown

NFKB in L6 cells (Fig. 5b). Furthermore, the LPS inhibitor polymyxin (which acts by sequestering LPS) prevented activation of the NFKB pathway by LPS (Fig $5 b$ ). We took advantage of the observation that TLR-4 signalling is critically dependent upon the adaptor protein TIRAP (TIRAP null mice have severely impaired TLR-4 mediated activation of the NFKB pathway in response to LPS) [36, 37] to demonstrate that a cell-permeable TIP prevented LPS activation of NFKB in L6 myotubes (Fig. 5c). We next addressed the role of TLR-4 in lipid-induced insulin resistance in L6 myotubes. A $6 \mathrm{~h}$ incubation of L6 myotubes with BSA-complexed palmitate substantially reduced insulin-stimulated 2-deoxyglucose uptake compared with control cells incubated in the presence of BSA alone (Fig. 6), without alterations in Il6 or Tnfa expression (data not shown). Importantly, in the presence of TIP, the capacity of palmitate to induce insulin resistance was sub- stantially decreased (Fig. 6, $p=0.004$ for palmitate/TIP vs palmitate). However, the reversal of insulin resistance was not complete, possibly due to the accumulation of the lipid metabolite diacylglycerol (but not ceramide), a proposed mediator of insulin resistance (data not shown). Palmitate also activated the NFKB pathway in L6 myotubes (Fig. 7a) and soleus muscle (Fig. 7c), and these effects were prevented by TIP, but not polymyxin, in L6 myotubes (Fig. 7b) and by TLR-4 deletion in soleus muscle (Fig. 7c). These data suggest activation of the NFKB pathway as a mechanism of TLR-4-induced insulin resistance in L6 cells. Indeed, the IKK inhibitor ASA reduced the capacity of fatty acids to induce insulin resistance (Fig. 8) and prevented activation of the NFKB pathway (data not shown).

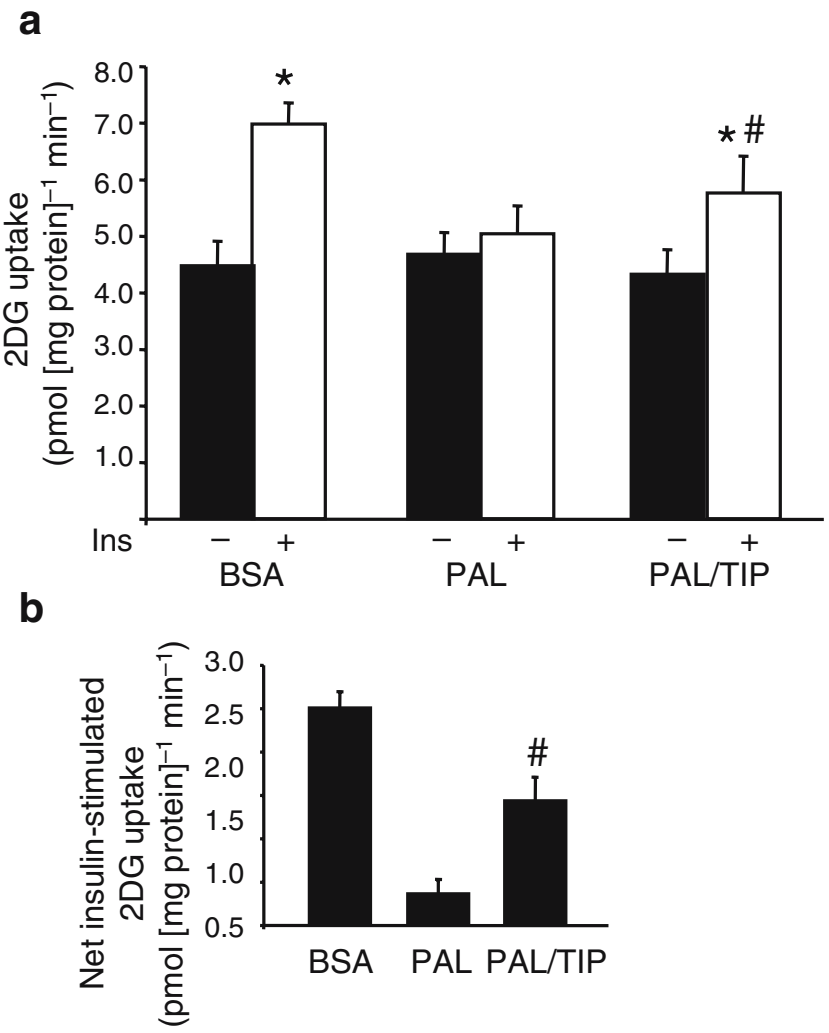

Fig. 6 Effects of TLR-4 inhibition on lipid-induced insulin resistance in L6 myotubes. L6 myotubes were exposed to BSA or $0.4 \mathrm{mmol} / 1$ palmitate/BSA (PAL) in the absence or presence of TIP for $6 \mathrm{~h}$. Subsequently, 2-deoxyglucose (2DG) uptake was assessed in the absence or presence of $100 \mathrm{nmol} / \mathrm{l}$ insulin. a 2-deoxyglucose uptake in the absence $(-)$ or presence $(+)$ of insulin (Ins). b Net insulinstimulated 2-deoxyglucose uptake. This was calculated as 2-deoxyglucose uptake in the presence of insulin minus 2-deoxyglucose uptake in the absence of insulin. There were no significant differences in basal 2-deoxyglucose uptake under any of the treatment conditions tested. *Significant difference $(p<0.05)$ between the insulin treated groups and the corresponding non-insulin treated control group. ${ }^{~ \#}$ Significant difference $(p<0.05)$ between PAL/TIP and PAL and BSA. Results are presented as means \pm SE of four independent experiments performed in triplicate 
a

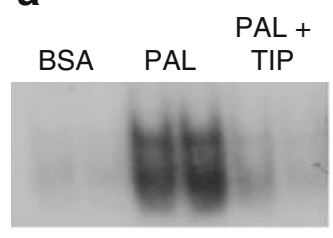

b BSA PAL LPS

PAL + Polymyxin

LPS +

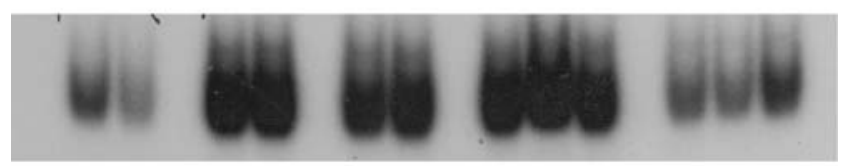

C

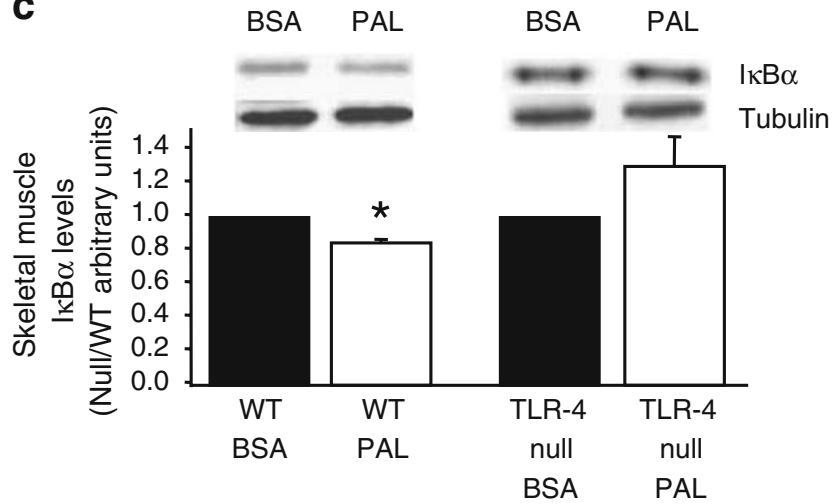

Fig. 7 Effects of palmitate on TLR-4-induced activation of the NFKB pathway in L6 myotubes and mouse skeletal muscle. a L6 myotubes were exposed to BSA, BSA/palmitate (PAL), or BSA/palmitate plus TIRAP inhibitor peptide (TIP) for $6 \mathrm{~h}$. Subsequently, nuclear extracts were prepared and DNA binding of NFKB was determined. Note that TIP was added to cells $1 \mathrm{~h}$ prior to the addition of palmitate. Representative EMSAs of three independent experiments for each condition run in duplicate are shown. b L6 myotubes were exposed to BSA, BSA/palmitate (PAL), LPS, BSA/palmitate plus polymyxin, or LPS plus polymyxin for $2 \mathrm{~h}$ (LPS) or $6 \mathrm{~h}$ (palmitate). Subsequently, nuclear extracts were prepared and DNA binding of NFKB was determined. Representative EMSAs of two independent experiments run in duplicate or triplicate are shown. c Soleus muscle from wildtype (WT) or TLR-4 null mice was exposed to BSA/palmitate (PAL) for $5 \mathrm{~h}$. The contralateral soleus muscle from the same animal was exposed to BSA for a similar length of time. For each condition in each experiment, muscles from three animals were used and pooled after exposures for analysis of $\operatorname{I\kappa B} \alpha$ and tubulin production by immunoblot. Representative autoradiographs are shown. Quantification of the complete data set is shown below the autoradiograph. For the purposes of quantification, I $\mathrm{K} \mathrm{B} \alpha$ values were normalised to tubulin values, and levels of $\operatorname{I\kappa B} \alpha$ in control skeletal muscle were arbitrarily set to a value of 1 . ${ }^{*}$ Significant difference $(p<0.05)$ vs wildtype BSA. $n=9$ animals/group. Results are presented as means $\pm \mathrm{SE}$

TLR-4 null mice on a high-fat diet have reduced adiposity and hepatic steatosis compared with wild-type controls Given the close association between hyperlipidaemia, inflammation and insulin resistance in obesity, one possibility is that activation of TLR-4 may be a mechanism that contributes to insulin resistance in obesity. To investigate this, TLR-4 null mice and wild-type controls were placed on a high-fat diet for
21 weeks. Adiposity, weight gain and hepatic steatosis were all reduced in TLR-4 null mice on a high-fat diet compared with wild-type controls fed the same diet (Table 1). However, the degree of insulin resistance was similar in knockout and wild-type controls (Fig. 9). There was a small, but significant, decrease in high-fat food intake in TLR-4 null mice compared with wild-type controls $[\sim 5 \%, 47.3 \pm 0.4$ vs $49.8 \pm 0.4 \mathrm{~kJ} /$ day $(11.3 \pm 0.1 \mathrm{vs} 11.9 \pm 0.1 \mathrm{kcal} /$ day $), p<0.05]$. This is unlikely to explain the differences in body weight gain $(\sim 25 \%, 16.5 \pm 3.9$ vs $21.6 \pm 2.2 \mathrm{~g})$, a conclusion supported by the study of Johnson et al. [38].

\section{Discussion}

The major goal of the current study was to assess a potential role for TLR-4, an important element of the innate immune system, in skeletal muscle insulin resistance induced by lipids and obesity. The motivation for these studies came
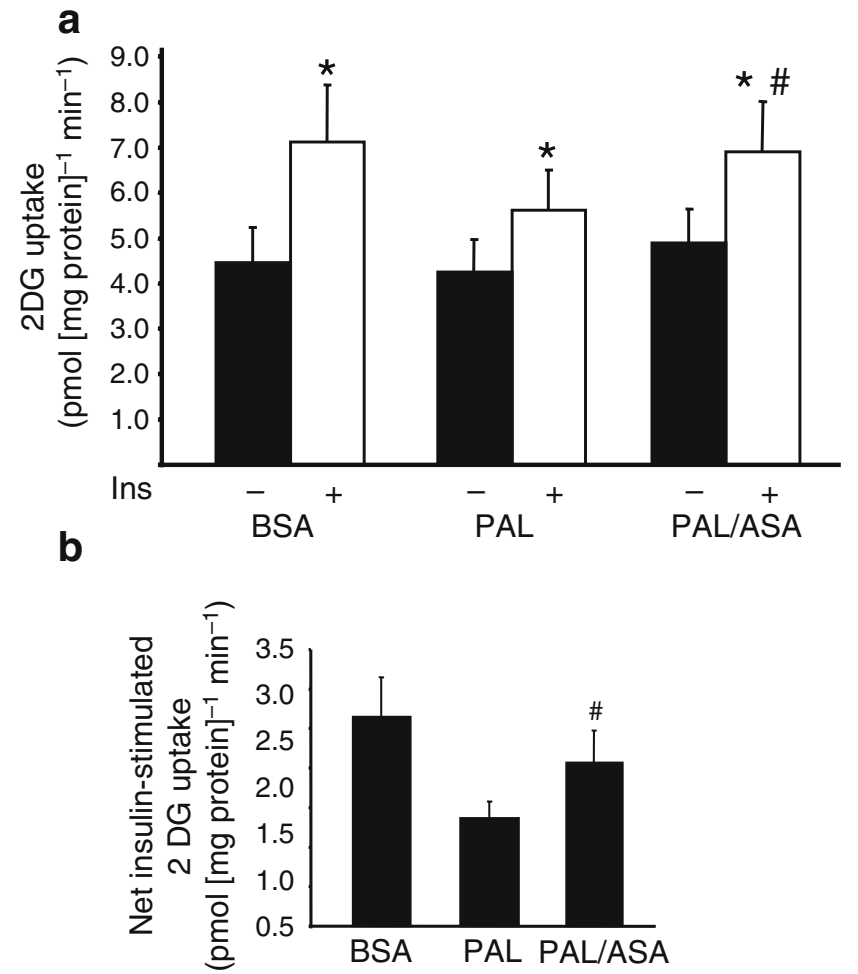

Fig. 8 Effects of the IKK inhibitor acetylsalicylate on lipid-induced insulin resistance in L6 myotubes. L6 myotubes were exposed to BSA, palmitate/BSA (PAL), or palmitate/BSA plus $5 \mathrm{mmol} / 1$ acetylsalicylate (PAL/ASA) for $6 \mathrm{~h}$. Subsequently, 2-deoxyglucose (2DG) uptake was assessed in the absence or presence of $100 \mathrm{nmol} / \mathrm{linsulin}$. a 2-Deoxyglucose uptake in the absence (-) or presence $(+)$ of insulin. b Net insulin-stimulated 2-deoxyglucose uptake. ${ }^{*}$ Significant difference $(p<0.05)$ between the insulin treated groups and the corresponding non-insulin treated control group. " Significant difference $(p<0.05)$ between PAL/ASA and PAL. Data represent the means $\pm \mathrm{SE}$ of at least three independent experiments performed in triplicate 
Table 1 Metabolic variables of TLR-4 null and wild-type mice on a standard chow or high-fat diet

\begin{tabular}{lllll}
\hline & Wild-type (SC) & Wild-type (HF) & TLR-4 (SC) & TLR-4 null (HF) \\
\hline Body weight gain (g) & $7.7 \pm 1.5$ & $21.6 \pm 2.2^{\mathrm{a}}$ & $7.2 \pm 1.0$ & $16.5 \pm 3.9^{\mathrm{a}, \mathrm{b}}$ \\
Liver weight (g) & $1.32 \pm 0.04$ & $1.60 \pm 0.10^{\mathrm{a}}$ & $1.23 \pm 0.03$ & $1.17 \pm 0.05^{\mathrm{b}}$ \\
Visceral adipose weight (g) & $1.19 \pm 0.15$ & $4.19 \pm 0.10^{\mathrm{a}}$ & $0.98 \pm 0.22$ & $3.54 \pm 0.28^{\mathrm{a}, \mathrm{b}}$ \\
Liver TG (mg/mg tissue) & $1.48 \pm 0.17$ & $2.85 \pm 0.32^{\mathrm{a}}$ & $1.87 \pm 0.30$ & $1.49 \pm 0.24^{\mathrm{b}}$ \\
Muscle TG (mg/mg tissue) & $0.7 \pm 0.1$ & $4.2 \pm 0.1^{\mathrm{a}}$ & $0.6 \pm 0.1$ & $3.3 \pm 0.4^{\mathrm{a}}$ \\
Plasma TG (mg/ml) & $18.6 \pm 2.3$ & $27.7 \pm 2.2^{\mathrm{a}}$ & $17.4 \pm 2.6$ & $23.1 \pm 2.4$ \\
Plasma glucose (mmol/l) & $5.5 \pm 0.2$ & $7.1 \pm 0.5^{\mathrm{a}}$ & $5.5 \pm 0.4$ & $6.9 \pm 0.7^{\mathrm{a}}$ \\
Plasma insulin (pmol/l) & $3.6 \pm 0.24$ & $5.4 \pm 1.26^{\mathrm{a}}$ & $1.2 \pm 0.12$ & $4.8 \pm 1.2^{\mathrm{a}}$ \\
\hline
\end{tabular}

Wild-type and TLR-4 null mice were fed a standard chow (SC) or high-fat (HF) diet for 21 weeks, during which time weight gain was monitored. Subsequently, animals were fasted overnight and killed. $n=$ a minimum 5 (range 5-15)

${ }^{a}$ Significant difference between the HF groups and corresponding SC group

${ }^{\mathrm{b}}$ Significant difference between the TLR-4 null HF group and wild-type HF group

TG, triacylglycerol

from increasing evidence that increased activity of the innate immune system may play a role in the pathogenesis of insulin resistance. Our studies have generated a number of novel observations. We demonstrate that (1) TLR-4 is produced in skeletal muscle and is activated by lipids; (2) inhibition or absence of skeletal muscle TLR-4 reduces the capacity of lipids to induce insulin resistance in vitro and in vivo; (3) the NFKB pathway is a mediator of the detrimental effects of TLR-4 activation on insulin action in skeletal muscle; and (4) the weight gain, adiposity, liver weight and steatosis induced by a high-fat diet is reduced in TLR-4deficient mice.

In previous studies [13] we demonstrated that the acute (6 h) exposure of L6 myotubes to palmitate induces insulin resistance, in agreement with numerous studies that have demonstrated the induction of insulin resistance in vitro and in vivo in response to a period of acute hyperlipidaemia [11, 13, 31, 39-41]. In the current study we demonstrate that inhibition or deletion of TLR-4 confers protection against the deleterious effects of lipids on skeletal muscle insulin sensitivity when L6 myotubes are exposed to

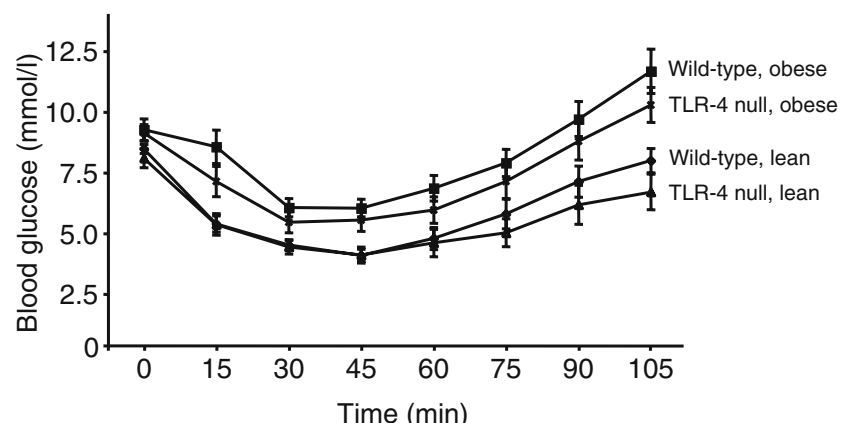

Fig. 9 Insulin tolerance in lean and obese TLR-4 null and wild-type mice. Wild-type and TLR-4 null mice were fed a standard chow or high-fat diet for 21 weeks. Subsequently, all mice underwent insulin tolerance tests as described in Methods. Results are presented as the means \pm SE for a minimum of ten animals in each group saturated fatty acids in vitro and when mice are exposed to a lipid infusion in vivo. In vivo, the protective effects of TLR-4 deletion were not related to alterations in skeletal muscle inflammatory gene expression, total GLUT4 or $\mathrm{I} \kappa \mathrm{B} \alpha$ levels. However, improvements in insulin sensitivity were associated with increased IRS-1-associated PI3-kinase activity and Akt phosphorylation. Our observations are in good agreement with the recent study of Shi et al. [17], which also demonstrated a reduced capacity of lipids to induce insulin resistance in TLR-4 null mice compared with control mice. However, the Shi et al. study did not directly investigate the potential role of skeletal muscle TLR-4 in this phenotype, concentrating instead on adipose tissue TLR-4. In this respect our study strongly suggests a direct role for skeletal muscle TLR-4 in the induction of insulin resistance by lipids. Indeed, this hypothesis is supported by the apparent lack of effect of lipid infusions on plasma levels of adipose-derived factors that could indirectly alter skeletal muscle insulin sensitivity, such as IL-6, TNF- $\alpha$, or resistin. It should also be noted that inhibition or the absence of TLR-4 was not sufficient to completely block the effects of lipids on skeletal muscle insulin action. This demonstrates that other mechanisms of lipid-induced insulin resistance operate in the absence of TLR-4 activity. In this regard there is strong evidence that intermediate lipid metabolites, such as diacylglycerol, ceramide and long chain acyl CoAs, mediate the detrimental effects of lipids on insulin action [39-46]. Indeed, diacylglycerol, but not ceramide, is increased in L6 myotubes after a $6 \mathrm{~h}$ incubation with palmitate (data not shown). Furthermore, a role for TLR-2 has recently been implicated in lipidinduced insulin resistance in skeletal muscle [47].

The NFKB pathway in skeletal muscle is activated by lipids in rodents [8] and humans [11]. Furthermore, lipidinduced skeletal muscle insulin resistance is decreased by inhibitors of the NFKB pathway $[10,12,13]$, while mice with a haplo-insufficiency of IKK $\beta$ [12] are protected 
against the effects of lipids on skeletal muscle insulin action. The NFKB pathway is a proximal target of TLR-4 activity, and saturated fatty acids activate TLR-4 in RAW 264.7 macrophages $[27,28]$. Thus, one mechanism by which reduced TLR-4 function may protect against the effects of lipids on insulin action is to decrease or prevent activation of the NFKB pathway. The current study presents pharmacological and genetic evidence in support of this hypothesis. Thus, TLR-4 signalling is inhibited by blocking the association of the adaptor molecule TIRAP with the TLR receptor [37]. This was accomplished in the current study by use of a cell-permeable TIP [37], which blocked the activation of NFKB by palmitate or LPS. Furthermore, the IKK $\beta$ inhibitor ASA (which therefore acts downstream of TLR-4) improved palmitate-induced insulin sensitivity and inhibited activation of the NFKB pathway (data not shown), demonstrating that lipid-induced activation of the $\mathrm{NF} \kappa \mathrm{B}$ pathway and insulin resistance are causally related, as reported by previous studies $[12,13]$. Genetic evidence comes from our experiments in mice lacking TLR-4 [23, 48]. Thus, muscles isolated from TLR-4 null mice did not respond to palmitate, as assessed by the levels of $I \kappa B \alpha$, while skeletal muscle I $\mathrm{K} \alpha \alpha$ levels from animals expressing $T l r-4$ were reduced after exposure to palmitate. It is presently unclear how activation of the NFKB pathway by lipids induces insulin resistance in skeletal muscle. It has been proposed that increased IKK $\beta$ activity can inhibit insulin-stimulated PI3-kinase activity, a hypothesis supported by the capacity of lipids to decrease, and salicylates to increase, insulin-stimulated PI3-kinase activity and Akt phosphorylation $[12,13]$. In this respect, the capacity of insulin to stimulate PI3-kinase activity and Akt phosphorylation to a greater extent in lipid-infused TLR-4 null mice than in control mice would support this hypothesis. Others have proposed that increased expression of NF $\mathrm{KB}$-dependent genes, such as Tnfa or Il6, may indirectly mediate the effects of lipids on insulin action. However, in the current study we did not observe increases in the expression of either of these genes in skeletal muscle in response to lipids.

There is a growing body of evidence in support of a role for activation of inflammatory pathways/the innate immune system in the development of insulin resistance in obesity. An obvious candidate mechanism for the initiating event leading to the activation of inflammatory pathways is dyslipidaemia. From this perspective the data in the current study and in others demonstrating activation of the NFKB pathway by acute hyperlipidaemia $[8,11,13]$ support this hypothesis. In the current study the absence of TLR-4 conferred partial protection against the development of obesity and steatosis, but not insulin resistance, in response to a high-fat diet. The observations of decreased weight gain and adiposity are in good agreement with the study of Johnson et al. [38], who report decreased body fat and weight gain with age in three strains of mice in which TLR4 is absent. Importantly, these metabolic alterations were not due to differences in activity or the presence of bacteria, toxins or viruses [38]. Our observations on insulin action are in agreement with the study of Shi et al. [17], which demonstrated increased insulin sensitivity on a high-fat diet in female, but not male, TLR-4 null mice (mice generated by the Akira group [49]), and the study of Poggi et al. [50] in $\mathrm{C} 3 \mathrm{H} / \mathrm{HeJ}$ mice with a spontaneous mutation in the $T l r-4$ gene [23]. Together, these studies make clear that caution should be used when discussing the potential role of TLR-4 in the development of insulin resistance in obesity (or type 2 diabetes). The metabolic profile of obesity is markedly more complicated then that of hyperlipidaemia alone. Alterations in tissue or circulating levels of acute phase reactants, interleukins and adipocytokines may all contribute, or indeed be primary, to the initiation and/or propagation of the inflammatory response and the development of insulin resistance in obesity. Clearly, this complex but vitally important area requires substantially more investigation.

Acknowledgements This work was supported by an American Diabetes Association Research Award (no. 1-05-RA-89) and a National Institutes of Health (NIH) grant (no. RO1 DK058855) (both to R. M. O'Doherty). B. A. Bhatt and M. S. Radin were supported by NIH grant no. T32-DK07052 (Research Training in Diabetes and Endocrinology).

Duality of interest The authors declare that there is no duality of interest associated with this manuscript.

\section{References}

1. Arkan MC, Hevener AL, Greten FR et al (2005) IKK-beta links inflammation to obesity-induced insulin resistance. Nat Med 11:191-198

2. Kanda H, Tateya S, Tamori Y et al (2006) MCP-1 contributes to macrophage infiltration into adipose tissue, insulin resistance, and hepatic steatosis in obesity. J Clin Invest 116:1494-1505

3. Weisberg SP, Hunter D, Huber R et al (2006) CCR2 modulates inflammatory and metabolic effects of high-fat feeding. $\mathrm{J}$ Clin Invest 116:115-124

4. Weisberg SP, McCann D, Desai M, Rosenbaum M, Leibel RL, Ferrante AW Jr (2003) Obesity is associated with macrophage accumulation in adipose tissue. J Clin Invest 112:1796-1808

5. Xu H, Barnes GT, Yang Q et al (2003) Chronic inflammation in fat plays a crucial role in the development of obesity-related insulin resistance. J Clin Invest 112:1821-1830

6. Kamei N, Tobe K, Suzuki R et al (2006) Overexpression of monocyte chemoattractant protein-1 in adipose tissues causes macrophage recruitment and insulin resistance. J Biol Chem 281:26602-26614

7. Di Gregorio GB, Yao-Borengasser A, Rasouli N et al (2005) Expression of CD68 and macrophage chemoattractant protein-1 genes in human adipose and muscle tissues: association with 
cytokine expression, insulin resistance, and reduction by pioglitazone. Diabetes 54:2305-2313

8. Bhatt B, Dube JJ, Dedousis N, Reider JA, O'Doherty RM (2006) Diet-induced obesity and acute hyperlipidemia reduce IkappaBalpha levels in rat skeletal muscle in a fiber-type dependent manner. Am J Physiol 290:R233-R240

9. Hirosumi J, Tuncman G, Chang L et al (2002) A central role for JNK in obesity and insulin resistance. Nature 420:333-336

10. Hundal RS, Petersen KF, Mayerson AB et al (2002) Mechanism by which high-dose aspirin improves glucose metabolism in type 2 diabetes. J Clin Invest 109:1321-1326

11. Itani SI, Ruderman NB, Schmieder F, Boden G (2002) Lipidinduced insulin resistance in human muscle is associated with changes in diacylglycerol, protein kinase C, and IkappaB-alpha. Diabetes 51:2005-2011

12. Kim JK, Kim YJ, Fillmore JJ et al (2001) Prevention of fat-induced insulin resistance by salicylate. J Clin Invest 108:437-446

13. Sinha S, Perdomo G, Brown NF, O'Doherty RM (2004) Fatty acid-induced insulin resistance in L6 myotubes is prevented by inhibition of activation and nuclear localization of NF-kB. J Biol Chem 279:41294-41301

14. Tripathy D, Mohanty P, Dhindsa S et al (2003) Elevation of free fatty acids induces inflammation and impairs vascular reactivity in healthy subjects. Diabetes 52:2882-2887

15. Yuan M, Konstantopoulos N, Lee J et al (2001) Reversal of obesity-and diet-induced insulin resistance with salicylates or targeted disruption of Ikkbeta. Science 293:1673-1677

16. Cai D, Yuan M, Frantz DF et al (2005) Local and systemic insulin resistance resulting from hepatic activation of IKK-beta and NFkappaB. Nat Med 11:183-190

17. Shi H, Kokoeva MV, Inouye K, Tzameli I, Yin H, Flier JS (2006) TLR4 links innate immunity and fatty acid-induced insulin resistance. J Clin Invest 116:3015-3025

18. Chambers JC, Eda S, Bassett P et al (2001) C-reactive protein, insulin resistance, central obesity, and coronary heart disease risk in Indian Asians from the United Kingdom compared with European whites. Circulation 104:145-150

19. Festa A, D'Agostino R Jr, Tracy RP, Haffner SM (2002) Elevated levels of acute-phase proteins and plasminogen activator inhibitor1 predict the development of type 2 diabetes: the Insulin Resistance Atherosclerosis Study. Diabetes 51:1131-1137

20. Grimble RF (2002) Inflammatory status and insulin resistance. Curr Opin Clin Nutr Metab Care 5:551-559

21. Beutler B, Rietschel ET (2003) Innate immune sensing and its roots: the story of endotoxin. Nat Rev Immunol 3:169-176

22. Takeda K, Kaisho T, Akira S (2003) Toll-like receptors. Annu Rev Immunol 21:335-376

23. Poltorak A, He X, Smirnova I et al (1998) Defective LPS signaling in $\mathrm{C} 3 \mathrm{H} / \mathrm{HeJ}$ and $\mathrm{C} 57 \mathrm{BL} / 10 \mathrm{ScCr}$ mice: mutations in Tlr4 gene. Science 282:2085-2088

24. O'Neill LA (2002) Toll-like receptor signal transduction and the tailoring of innate immunity: a role for Mal? Trends Immunol 23:296-300

25. Beutler B (2004) Inferences, questions and possibilities in Tolllike receptor signalling. Nature 430:257-263

26. Akira S, Takeda K (2004) Toll-like receptor signalling. Nat Rev Immunol 4:499-511

27. Lee JY, Sohn KH, Rhee SH, Hwang D (2001) Saturated fatty acids, but not unsaturated fatty acids, induce the expression of cyclooxygenase-2 mediated through Toll-like receptor 4. J Biol Chem 276:16683-16689

28. Lee JY, Ye J, Gao Z et al (2003) Reciprocal modulation of Tolllike receptor-4 signaling pathways involving MyD88 and phosphatidylinositol 3-kinase/AKT by saturated and polyunsaturated fatty acids. J Biol Chem 278:37041-37051
29. Lin Y, Lee H, Berg AH, Lisanti MP, Shapiro L, Scherer PE (2000) The lipopolysaccharide-activated toll-like receptor (TLR)-4 induces synthesis of the closely related receptor TLR-2 in adipocytes. J Biol Chem 275:24255-24263

30. Liu S, Gallo DJ, Green AM et al (2002) Role of toll-like receptors in changes in gene expression and NF-kappa B activation in mouse hepatocytes stimulated with lipopolysaccharide. Infect Immun 70:3433-3442

31. Boden G, Lebed B, Schatz M, Homko C, Lemieux S (2001) Effects of acute changes of plasma free fatty acids on intramyocellular fat content and insulin resistance in healthy subjects. Diabetes 50:1612-1617

32. Bachmann OP, Dahl DB, Brechtel K et al (2001) Effects of intravenous and dietary lipid challenge on intramyocellular lipid content and the relation with insulin sensitivity in humans. Diabetes 50:2579-2584

33. Krssak M, Falk Petersen K, Dresner A et al (1999) Intramyocellular lipid concentrations are correlated with insulin sensitivity in humans: a $1 \mathrm{H}$ NMR spectroscopy study. Diabetologia 42:113-116

34. O'Doherty RM, Halseth AE, Granner DK, Bracy DP, Wasserman DH (1998) Analysis of insulin-stimulated skeletal muscle glucose uptake in conscious rat using isotopic glucose analogs. Am J Physiol 274:E287-E296

35. Huang W, Dedousis N, Bandi A, Lopaschuk GD, O'Doherty RM (2006) Liver triglyceride secretion and lipid oxidative metabolism are rapidly altered by leptin in vivo. Endocrinology 147:1480 1487

36. Horng T, Barton GM, Flavell RA, Medzhitov R (2002) The adaptor molecule TIRAP provides signalling specificity for Tolllike receptors. Nature 420:329-333

37. Horng T, Barton GM, Medzhitov R (2001) TIRAP: an adapter molecule in the Toll signaling pathway. Nat Immunol 2:835841

38. Johnson GB, Riggs BL, Platt JL (2004) A genetic basis for the "Adonis" phenotype of low adiposity and strong bones. FASEB J 18:1282-1284

39. Chavez JA, Knotts TA, Wang LP et al (2003) A role for ceramide, but not diacylglycerol, in the antagonism of insulin signal transduction by saturated fatty acids. J Biol Chem 278:10297-10303

40. Chalkley SM, Hettiarachchi M, Chisholm DJ, Kraegen EW (1998) Five-hour fatty acid elevation increases muscle lipids and impairs glycogen synthesis in the rat. Metabolism 47:11211126

41. Griffin ME, Marcucci MJ, Cline GW et al (1999) Free fatty acidinduced insulin resistance is associated with activation of protein kinase $\mathrm{C}$ theta and alterations in the insulin signaling cascade. Diabetes 48:1270-1274

42. Adams JM 2nd, Pratipanawatr T, Berria R et al (2004) Ceramide content is increased in skeletal muscle from obese insulin-resistant humans. Diabetes 53:25-31

43. Boden G, Shulman GI (2002) Free fatty acids in obesity and type 2 diabetes: defining their role in the development of insulin resistance and beta-cell dysfunction. Eur J Clin Invest 32(Suppl 3):1423

44. Perdomo G, Commerford SR, Richard AM et al (2004) Increased beta-oxidation in muscle cells enhances insulin-stimulated glucose metabolism and protects against fatty acid-induced insulin resistance despite intramyocellular lipid accumulation. J Biol Chem 279:27177-27186

45. Petersen KF, Shulman GI (2002) Pathogenesis of skeletal muscle insulin resistance in type 2 diabetes mellitus. Am J Cardiol 90:11G-18G

46. Yu C, Chen Y, Cline GW et al (2002) Mechanism by which fatty acids inhibit insulin activation of insulin receptor substrate-1 
(IRS-1)-associated phosphatidylinositol 3-kinase activity in muscle. J Biol Chem 277:50230-50236

47. Senn JJ (2006) Toll-like receptor-2 is essential for the development of palmitate-induced insulin resistance in myotubes. J Biol Chem 281:26865-26875

48. Coutinho A, Forni L, Melchers F, Watanabe T (1977) Genetic defect in responsiveness to the $\mathrm{B}$ cell mitogen lipopolysaccharide. Eur J Immunol 7:325-328
49. Hoshino K, Takeuchi O, Kawai T et al (1999) Cutting edge: Tolllike receptor 4 (TLR4)-deficient mice are hyporesponsive to lipopolysaccharide: evidence for TLR4 as the Lps gene product. J Immunol 162:3749-3752

50. Poggi M, Bastelica D, Gual P et al (2007) C3H/HeJ mice carrying a toll-like receptor 4 mutation are protected against the development of insulin resistance in white adipose tissue in response to a high-fat diet. Diabetologia 50:1267-1276 